\title{
Functional summary statistics for the Johnson-Mehl model
}

\author{
February 22, 2013
}

\author{
Jesper Møller and Mohammad Ghorbani \\ Department of Mathematical Sciences, Aalborg University
}

\begin{abstract}
The Johnson-Mehl germination-growth model is a spatio-temporal point process model which among other things have been used for the description of neurotransmitters datasets. However, for such datasets parametric Johnson-Mehl models fitted by maximum likelihood have yet not been evaluated by means of functional summary statistics. This paper therefore invents four functional summary statistics adapted to the Johnson-Mehl model, with two of them based on the second-order properties and the other two on the nuclei-boundary distances for the associated Johnson-Mehl tessellation. The functional summary statistics theoretical properties are investigated, non-parametric estimators are suggested, and their usefulness for model checking is examined in a simulation study. The functional summary statistics are also used for checking fitted parametric Johnson-Mehl models for a neurotransmitters dataset.
\end{abstract}

Key words: germination-growth process, model checking, neurotransmitters, pair correlation function, spatio-temporal point process, tessellation, typical cell.

\section{Introduction}

This paper concerns statistical inference for the Johnson-Mehl germination-growth process in the $d$ dimensional Euclidean space $\mathbb{R}^{d}$, with a focus on model fitting and checking. The case $d=1$ is of special interest for modelling e.g. neurotransmitters datasets, but many of our results apply for $d \geq 1$ as well. Section 1.1 recalls the definition of the Johnson-Mehl model, Section 1.2 reviews the literature and the applications of the model, and Section 1.3 states our main objectives and the outline of the paper.

\subsection{The Johnson-Mehl model}

The Johnson-Mehl germination-growth process results by a dependent thinning of a space-time Poisson process as follows. 
We start with a primary space-time Poisson process $\Phi \equiv\left\{\left(x_{1}, t_{1}\right),\left(x_{2}, t_{2}\right), \ldots\right\} \subset \mathbb{R}^{d} \times[0, \infty)$ with intensity measure $\mathrm{d} x \mathcal{K}(\mathrm{d} t)$, where $\mathrm{d} x$ denotes Lebesgue measure on $\mathbb{R}^{d}$ and $\mathcal{K}$ is a non-zero locally finite measure on $[0, \infty)$. For $\left(x_{i}, t_{i}\right) \in \Phi$, we call $\left(x_{i}, t_{i}\right)$ an event and think of $x_{i}$ as a nucleus (or germ) which starts to grow at time $t_{i}$ with a constant speed $v>0$ in all directions, unless $x_{i}$ has already been reached by another nucleus $x_{j}$ which started to grow at a time $t_{j}<t_{i}$ (with speed $v$ in all directions); in the latter case we say that $\left(x_{i}, t_{i}\right)$ has been thinned. Let

$$
T_{i}(y) \equiv T\left(\left(x_{i}, t_{i}\right), y\right)=t_{i}+\left\|x_{i}-y\right\| / v
$$

be the time that $x_{i}$ reaches a point $y \in \mathbb{R}^{d}$. Then the unthinned events form a secondary space-time point process, namely the Johnson-Mehl germination-growth process (or shortly Johnson-Mehl process) given by

$$
\Psi=\left\{\left(x_{i}, t_{i}\right) \in \Phi: t_{i} \leq T_{j}\left(x_{i}\right) \text { for all }\left(x_{j}, t_{j}\right) \in \Phi \text { with } t_{j}<t_{i}\right\}
$$

Moreover, the growth along the ray of any growing nucleus stops when it reaches another growing ray. Thereby a tessellation of $\mathbb{R}^{d}$ with cells

$$
C_{i}=\left\{y \in \mathbb{R}^{d}: T_{i}(y) \leq T_{j}(y) \text { for all } j \neq i \text { with }\left(x_{j}, t_{j}\right) \in \Psi\right\}, \quad\left(x_{i}, t_{i}\right) \in \Psi
$$

is created. This is called the Johnson-Mehl tessellation, see Johnson \& Mehl (1939), Stoyan, Kendall \& Mecke (1995), Okabe, Boots, Sugihara \& Chiu (2000), and the references therein. In the special case where $\mathcal{K}$ is concentrated at time 0 , the Johnson-Mehl tessellation is just a Voronoi tessellation (Møller 1994; Okabe et al. 2000).

\subsection{Applications and literature}

Applications of the Johnson-Mehl model for $d=2$ and $d=3$ can be found in crystal growth (Kolmogorov 1937; Johnson \& Mehl 1939), microstructural evolution in thin films (see Frost, Thompson \& Walton (1992) and the references therein), plant ecology (Kenkel 1990), and socio-economic cellular network (Boots, Okabe \& Thomas 2003). Biological applications of the model for $d=1$ include DNA replication (Vanderbei \& Shepp 1988; Cowan, Chiu \& Holst 1995), release of neurotransmitters at neuromuscular synapses (Quine \& Robinson 1990; Quine \& Robinson 1992; Bennett \& Robinson 1990; Chiu, Quine \& Stewart 2000; Molchanov \& Chiu 2000; Chiu, Molchanov \& Quine 2003), cell biology (Wolk 1975), and lung carcinoma (Kayser \& Stute 1989). The model has been well studied from a probabilistic point of view, with the pioneering work by Kolmogorov (1937) and Johnson \& Mehl (1939), and followed by Meijering (1953), Gilbert (1962), Miles (1972), Horálek (1988, 1990) and Møller (1992, 1995). Statistical 
analysis for the model has mainly been studied in the above-mentioned papers by Chiu and coworkers, with most attention to the case $d=1$ where maximum likelihood based inference is in fact feasible as discussed in Section 2.4.

\subsection{Aim and outline}

To the best of our knowledge, though functional summary statistics play a fundamental role for analyzing spatial and spatio-temporal point patterns (see e.g. Gabriel \& Diggle (2009) and Møller \& Ghorbani (2012)), this is the first paper introducing functional summary statistics for the Johnson-Mehl model.

In the theory part (Sections 2-3), we consider the general case $d \geq 1$, since applications occur for $d=1,2,3$ (cf. Section 1.2) and many ideas and results can easily be stated. In the application part (Section 4$), d=1$.

Section 2 provides the needed background material for this paper. Section 3 introduces our functional summary statistics. First, two functional summary statistics based on the pair correlation function and with some relation to the space-time inhomogeneous $K$-function (Gabriel \& Diggle 2009) are invented. Second, two functional summary statistics based on the nuclei-boundary distances for the Johnson-Mehl tessellation are developed. The theoretical properties and non-parametric estimation of the functional summary statistics are discussed. Section 4 presents the results of a simulation study when applying the functional summary statistics for model checking in cases where a Johnson-Mehl model is correctly or incorrectly specified. Moreover, for the same neurotransmitters dataset as analyzed in Chiu et al. (2003), we check parametric Johnson-Mehl models estimated by maximum likelihood. Finally, technical details are deferred to Appendixes A-D.

\section{Preliminaries}

This section introduces some notation, concepts, and parametric models used in the sequel.

\subsection{Independence}

Let $\Omega=\mathbb{R}^{d} \times[0, \infty)$ be the space-time domain, and for $A \subseteq \Omega$, let $\Phi_{A}=\Phi \cap A$ and $\Psi_{A}=\Psi \cap A$. For $(x, t) \in \Omega$, denote

$$
C(x, t)=\{(y, u) \in \Omega: T((y, u), x) \leq t\}
$$


which is a cone consisting of those $(y, u) \in \Omega$ where $y$ reaches $x$ before or at time $t$ when $y$ starts to grow at time $u$ with speed $v$ in all directions. Hence, with probability one,

$$
\Psi=\left\{(x, t) \in \Phi: \Phi_{C(x, t)}=\{(x, t)\}\right\}
$$

For $A \subseteq \Omega$, define

$$
\bar{A}=\bigcup_{(x, t) \in A} C(x, t)=\{(y, u) \in \Omega: T((y, u), x)<t \text { for some }(x, t) \in A\} .
$$

By (2), $\Psi_{A}$ depends on $\Phi$ only through $\Phi_{\bar{A}}$. Therefore, for any Borel sets $A, B \subseteq \Omega$,

$$
\Psi_{A} \text { and } \Psi_{B} \text { are independent if } \bar{A} \cap \bar{B}=\emptyset
$$

since $\Phi_{\bar{A}}$ and $\Phi_{\bar{B}}$ are independent when $\bar{A} \cap \bar{B}=\emptyset$. Consequently, we say that $\left(x_{i}, t_{i}\right),\left(x_{j}, t_{j}\right) \in \Psi$ are independent events if $C\left(x_{i}, t_{i}\right) \cap C\left(x_{j}, t_{j}\right)=\emptyset$, that is, $v\left(t_{i}+t_{j}\right)<\left\|x_{i}-x_{j}\right\|$.

\subsection{Intensity}

Henceforth we assume that $\mathcal{K}$ is absolutely continuous w.r.t. Lebsgue measure on $[0, \infty)$, with density $\kappa$. It is also assumed that $\kappa$ is chosen such that all integrals to follow exit. This will be the case if e.g. $\kappa$ is piecewise continuous.

Note that $\Psi$ is stationary and isotropic in space, but inhomogeneous in time. Hence its intensity function depends only on time and is given by

$$
\rho(t)=\mathrm{P}\left(\Phi_{C(0, t)}=\emptyset\right) \kappa(t)=\exp \left(-\iint_{C(0, t)} \kappa(s) \mathrm{d} x \mathrm{~d} s\right) \kappa(t)
$$

as obtained from (2) and the Slivnyak-Mecke theorem (Mecke 1967; Møller \& Waagepetersen 2004). This reduces to

$$
\rho(t)=\exp \left(-\int_{0}^{t} \omega_{d}[v(t-s)]^{d} \kappa(s) \mathrm{d} s\right) \kappa(t)
$$

where $\omega_{d}=\pi^{d / 2} / \Gamma(1+d / 2)$ is the volume of the unit ball in $\mathbb{R}^{d}$. Indeed, $\rho$ is non-constant no matter the choice of $\kappa$. 


\section{$2.3 \quad$ Parametric models}

We are particularly interested in the following parametric models.

$$
\begin{aligned}
& \text { M1: } \kappa(t)=\alpha t^{\beta-1} \quad \text { where } \alpha>0, \beta>0 . \\
& \text { M2: } \kappa(t)=\frac{\alpha \gamma^{\beta}}{\Gamma(\beta)} t^{\beta-1} \exp (-\gamma t) \quad \text { where } \alpha>0, \beta>0, \gamma>0 .
\end{aligned}
$$

The models have been studied in Frost \& Thompson (1987), Horálek (1988,1990), Møller $(1992,1995)$, Chiu (1995), and the papers discussed in Section 4.2.1.

For M1, combining (4) and (5), we obtain

$$
\rho(t)=\exp \left(-\alpha \omega_{d} v^{d} t^{\beta+d} B(\beta, d+1)\right) \alpha t^{\beta-1}
$$

which is an un-normalized generalized gamma density with shape parameter $\beta$ and inverse scale parameter $\alpha$. For M2, $\kappa$ is an un-normalized gamma density, and combining (4) and (6), we obtain

$$
\begin{aligned}
\rho(t) & =\exp \left(-2 \alpha v\left(t \Gamma(t ; \beta, \gamma)-\frac{\beta}{\gamma} \Gamma(t ; \beta+1, \gamma)\right)\right) \frac{\alpha \gamma^{\beta}}{\Gamma(\beta)} t^{\beta-1} \exp (-\gamma t) & \text { if } d=1, \\
\rho(t) & =\exp \left(-\pi \alpha v^{2}\left(t^{2} \Gamma(t ; \beta, \gamma)-2 t \frac{\beta}{\gamma} \Gamma(t ; \beta+1, \gamma)+\frac{(\beta+1) \beta}{\gamma^{2}} \Gamma(t ; \beta+2, \gamma)\right)\right) & \\
& \times \frac{\alpha \gamma^{\beta}}{\Gamma(\beta)} t^{\beta-1} \exp (-\gamma t) & \text { if } d=2,
\end{aligned}
$$

and similar but more and more complicated formulae can be derived for $d \geq 3$. Here $\Gamma(x ; \beta, \gamma)$ denotes the cumulative distribution functions of a Gamma distribution with shape parameter $\beta$ and inverse scale parameter $\gamma$. Plots of $\rho$ under M1 and M2 are shown in Figure 1 where $d=1$; similar plots are obtained for $d=2$.

\subsection{Maximum likelihood}

Assuming the nuclei are observed within a bounded region $W \subset \mathbb{R}^{d}$, and ignoring edge effects due to thinning caused by the unobserved events with nuclei outside $W$, the unthinned events with nuclei within $W$ are given by

$$
\Psi_{W}^{\mathrm{app}}=\left\{\left(x_{i}, t_{i}\right) \in \Phi_{W \times[0, \infty)}: t_{i} \leq T_{j}\left(x_{i}\right) \text { for all }\left(x_{j}, t_{j}\right) \in \Phi_{W \times[0, \infty)} \text { with } t_{j}<t_{i}\right\} .
$$

The space-time point process $\Psi_{W}^{\text {app }}$ has conditional intensity function

$$
\lambda\left(x, t \mid \mathcal{H}_{t}\right)=\mathbf{1}\left[t \leq T_{i}(x) \text { for all }\left(x_{i}, t_{i}\right) \in \Psi_{W}^{\mathrm{app}} \text { with } t_{i}<t\right] \kappa(t), \quad(x, t) \in W \times[0, \infty),
$$



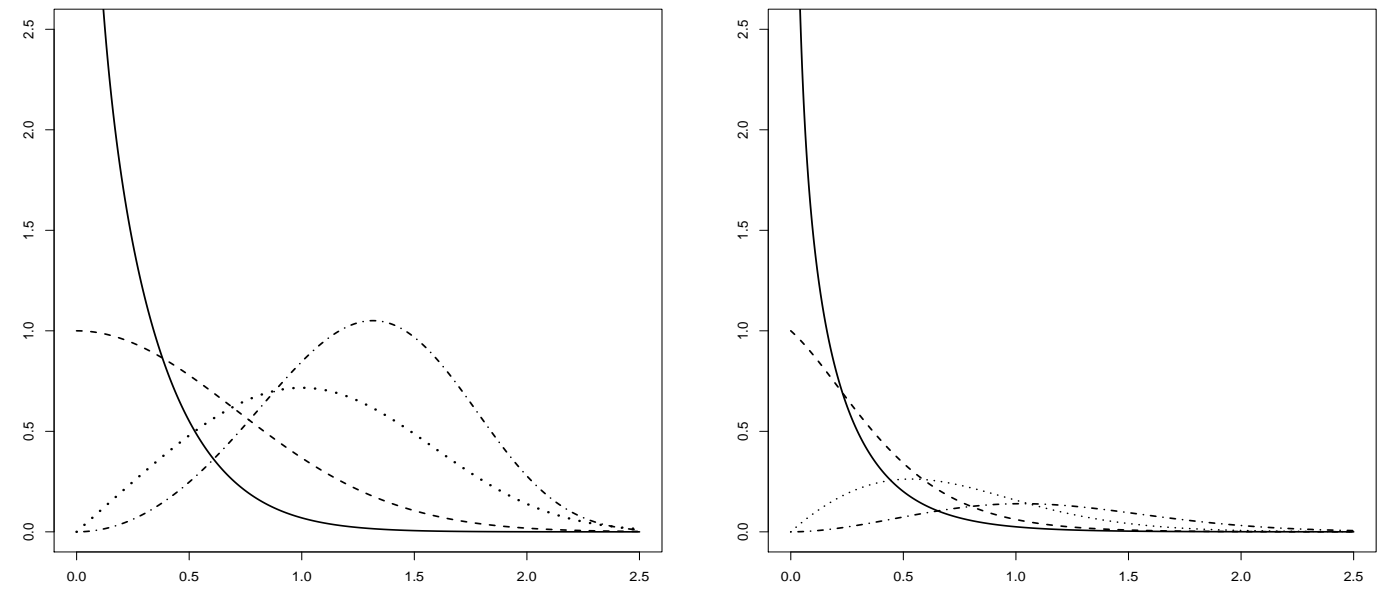

Figure 1: The intensity function $\rho(t)$ under M1 (left panel) and M2 (right panel) with $d=1, \alpha=\gamma=$ $v=1, \beta=0.5$ (solid line), $\beta=1$ (dashed line), $\beta=2$ (dotted line), and $\beta=3$ (dot-dashed line).

where $\mathbf{1}[\cdot]$ denotes the indicator function and $\mathcal{H}_{t}$ is the 'history' (i.e., the information about $\Psi_{W}^{\text {app }}$ up to but not including time $t$ ), cf. Daley \& Vere-Jones (2003).

Consider the problem of maximum likelihood estimation for $(v, \theta)$ when we have assumed a parametric model $\kappa_{\theta}$ for the function $\kappa$, e.g. $\theta=(\alpha, \beta) \in(0, \infty)^{2}$ in case of M1 or $\theta=(\alpha, \beta, \gamma) \in(0, \infty)^{3}$ in case of M2, and where $v$ and $\theta$ are variation independent. Suppose we observe a realization $\Psi_{W}^{\text {app }}=$ $\left\{\left(x_{1}, t_{1}\right), \ldots,\left(x_{n}, t_{n}\right)\right\}$ where $n<\infty$ and $t_{i} \neq t_{j}$ whenever $i \neq j$ (this is almost surely the case). By (11) and Section 7.2 in Daley \& Vere-Jones (2003), the likelihood function is

$$
\begin{aligned}
L(\theta, v) & =\mathbf{1}\left[t_{i}+\left\|x_{i}-x_{j}\right\| / v \geq t_{j} \text { whenever } t_{i}<t_{j}\right]\left[\prod_{i=1}^{n} \kappa_{\theta}\left(t_{i}\right)\right] \\
& \times \exp \left(-\iint_{W \times[0, \infty)} \mathbf{1}\left[t_{i}+\left\|x_{i}-x\right\| / v \geq t \text { whenever } t_{i}<t\right] \kappa_{\theta}(t) \mathrm{d} x \mathrm{~d} t\right) .
\end{aligned}
$$

See also Chiu et al. (2003). If $n \geq 2$, the maximum likelihood estimate (MLE) for $v$ is seen to be smallest pairwise relative distance

$$
\hat{v}=\min _{1 \leq i<j \leq n}\left\|x_{i}-x_{j}\right\| /\left|t_{i}-t_{j}\right|
$$

since $L(\theta, v)$ is an increasing function of $v \leq \hat{v}$, and it is zero for $v>\hat{v}$; if $n \leq 1$, then the MLE does not 
exist. Assuming $n \geq 2$, the profile likelihood $L(\theta)=L(\theta, \hat{v})$ is

$$
L(\theta)=\prod_{i=1}^{n} \kappa_{\theta}\left(t_{i}\right) \exp \left(-\int_{\hat{C}_{i}}\left(\int_{0}^{\hat{T}_{i}(x)} \kappa_{\theta}(t) \mathrm{d} t\right) \mathrm{d} x\right)
$$

where $\hat{T}_{i}(x)=t_{i}+\hat{v}\left\|x-x_{i}\right\|$ and $\hat{C}_{i}$ is the Johnson-Mehl cell as given by (1) when $v$ is replaced by $\hat{v}$, and $\Psi$ by $\left\{\left(x_{1}, t_{1}\right), \ldots,\left(x_{n}, t_{n}\right)\right\}$. When $d=1$, the integral in (12) may easily be evaluated, while numeric methods are needed for $d \geq 2$.

The extension to the case with independent copies of $\Psi_{W}^{\text {app }}$ (as considered in Section 4) is straightforward. In particular, the MLE for $v$ is simply the smallest pairwise relative distance obtained from the realizations of these independent copies.

\section{$3 \quad$ Functional summary statistics}

\subsection{Second-order analysis}

The natural staring point when characterizing the second-order properties of $\Psi$ is to consider the density of the second-order product for $\Psi$, denoted $\rho^{(2)}((x, s),(y, t))$ for $(x, s),(y, t) \in \Omega$, and called the second-order product density for $\Psi$, see e.g. Møller \& Ghorbani (2012) for a formal definition. Intuitively, $\rho^{(2)}((x, s),(y, t)) \mathrm{d} x \mathrm{~d} s \mathrm{~d} y \mathrm{~d} t$ is the probability of observing one event of $\Psi$ in an infinitesimally small region of volume $\mathrm{d} x \mathrm{~d} s$ around $(x, s)$ and another event of $\Psi$ in an infinitesimally small region of volume $\mathrm{d} y \mathrm{~d} t$ around $(y, t)$. Since $\Psi$ is stationary and isotropic in space and $\rho^{(2)}$ is a symmetric function, $\rho^{(2)}((x, s),(y, t))$ depends only on $x$ and $y$ through $r=\|x-y\|$, and it is symmetric w.r.t. $s$ and $t$, so we can write

$$
\rho^{(2)}((x, s),(y, t))=\rho_{0}^{(2)}(r, s, t)=\rho_{0}^{(2)}(r, t, s) .
$$

In Section 3.1.1, we determine $\rho_{0}^{(2)}$ and thereby the pair correlation function $g$ defined by

$$
g(r, t, s)=\frac{\rho_{0}^{(2)}(r, t, s)}{\rho(s) \rho(t)} \text { if } \rho(s) \rho(t)>0, \quad g(r, t, s)=0 \text { otherwise. }
$$

This is used in Section 3.1.2 for constructing functional second-order summary statistics.

\subsubsection{Pair correlation function}

Using (2) and the Slivnyak-Mecke formula (Mecke 1967; Møller \& Waagepetersen 2004) we see that

$$
\rho_{0}^{(2)}(r, s, t)=\kappa(s) \kappa(t) \mathbf{1}[r>v|s-t|] \exp \left(-\iint \kappa(u) \mathbf{1}[(z, u) \in C(x, s) \cup C(y, t)] \mathrm{d} z \mathrm{~d} u\right) .
$$


Let $b(x, r)$ denote the closed ball with center $x$ and radius $r>0$; set $b(x, r)=\emptyset$ if $r \leq 0$. For $u \geq 0$, let $V_{\cap}(r, s-u, t-u)$ denote the volume of the intersection $b(x, v(s-u)) \cap b(y, v(t-u))$ corresponding to the region in $\Omega$ of points $z$ born at time $u$ so that $z$ reaches $x$ before time $s$, and $y$ before time $t$, when growing with speed $v$ in all directions. Combining (4) and (13)-(14) we obtain

$$
\begin{aligned}
g(r, s, t) & =\mathbf{1}[v|s-t|<r<v(s+t)] \exp \left(-\int_{0}^{(s+t-r / v) / 2} \kappa(u) V_{\cap}(r, s-u, t-u) \mathrm{d} u\right) \\
& +\mathbf{1}[r \geq v(s+t)]
\end{aligned}
$$

if $\kappa(s)>0$ and $\kappa(t)>0$, and $g(r, t, s)=0$ otherwise. Thus $g(r, s, t) \leq 1$, meaning that $\Psi$ exhibits regularity. For $\kappa(s)>0$ and $\kappa(t)>0, g(r, s, t)$ as a function of $r$ is zero if $r \leq v|s-t|$; has a jump at $r=v|s-t|$ if and only if $\mathcal{K}([0,2 \min \{s, t\}])>0$; is non-decreasing and continuous if $r>v|s-t|$; and is one if $r \geq v(s+t)$, which is in accordance with our concept of independent events, cf. (3).

In order to calculate $V_{\cap}(\ldots)$ in (15), note that for $h \geq 0$ and $l>0$,

$$
V_{d}(l, h)=\frac{\pi^{d / 2} l^{d}}{2 \Gamma(1+d / 2)} I_{\left(2 l h-h^{2}\right) / l^{2}}((d+1) / 2,1 / 2)
$$

is the volume of a d-dimensional hyper-spherical cap of height $h$ and radius $l$ (Li 2011), where

$$
I_{c}(a, b)=\frac{1}{B(a, b)} \int_{0}^{c} u^{a-1}(1-u)^{b-1} \mathrm{~d} u \quad \text { with } B(a, b)=\frac{\Gamma(a) \Gamma(b)}{\Gamma(a+b)}
$$

is the regularized incomplete beta function. Thus, for $r>v|s-t|$ and $0 \leq u<(s+t-r / v) / 2$,

$$
\begin{aligned}
V_{\cap}(r, s-u, t-u) & =V_{d}\left(v(s-u), \frac{[v(t-u)]^{2}-(r-v(s-u))^{2}}{2 r}\right) \\
& +V_{d}\left(v(t-u), \frac{[v(s-u)]^{2}-(r-v(t-u))^{2}}{2 r}\right) .
\end{aligned}
$$

Particularly,

$$
V_{\cap}(r, s-u, t-u)=v(s+t-2 u)-r \quad \text { if } d=1
$$

while more complicated expressions are available for $d \geq 2$, see Appendix A.

Suppose that $v|s-t|<r<v(s+t)$, since this is the only case where it is not obvious what $g$ is, cf. (15). For simplicity, let $d=1$. For model M1, combining (5), (15), and (18), we obtain

$$
g(r, s, t)=\exp \left(-\frac{\alpha v}{\beta(\beta+1) 2^{\beta}}(s+t-r / v)^{\beta+1}\right) .
$$

For model M2, combining (6), (15), and (18), we obtain

$$
g(r, s, t)=\exp \left(-\alpha v\left(q \Gamma(q / 2 ; \beta, \gamma)-\frac{2 \beta}{\gamma} \Gamma(q / 2 ; \beta+1, \gamma)\right)\right),
$$

which is a strictly decreasing function of $q$, where $q=s+t-r / v$. 


\subsubsection{Functional second-order summary statistics}

Non-parametric estimates of the second-order properties of spatial and spatio-temporal point processes play a fundamental role for exploratory and explanatory analysis of spatial and spatio-temporal point patterns, see e.g. Gelfand, Diggle, Guttorp \& Fuentes (2010). However, we will avoid non-parametric estimation of the pair correlation function $g$, e.g. by extending the kernel estimators in Stoyan \& Stoyan (1996) from the spatial case to the space-time case, partly since these are much depending on the choice of bandwidth and partly because $g(r, s, t)$ is a three-dimensional function which is difficult to visualize.

In the spatial and space-time point process literature, second-order intensity-reweighted stationarity is often assumed, which in the space-time case means that the pair correlation function is only a function of $r$ and $t-s$. This property is then exploited for constructing so-called (inhomogeneous) $K$-functions, obtained by integrating the pair correlation function, and which are easy to estimate and use for exploratory purpuses and for model checking of fitted models (Baddeley, Møller \& Waagepetersen 2000; Gabriel \& Diggle 2009).

Since $\Psi$ is not second-order intensity-reweighted stationary, cf. (15) and (18), we will instead propose another way of constructing useful functional second-order summary statistics which have some relation to the inhomogeneous space-time $K$-function and which are much related to our setting of the JohnsonMehl model. We let $W \subset \mathbb{R}^{d}$ denote a bounded observation window of Lebesgue measure $|W|>0$, and assume that a realization of $\Psi_{W \times[0, \infty)}$ is observed. Moreover, we pretend that $v, \kappa$, and $\rho$ are known, though in practice, in all expressions to follow in this section, we may replace these parameters by maximum likelihood estimates (Section 2.4).

Now, for $R>0$, define

$$
K_{1}(R)=\mathrm{E} \sum_{i \neq j} \frac{\mathbf{1}\left[x_{i} \in W, v\left(t_{i}+t_{j}\right)<\left\|x_{i}-x_{j}\right\| \leq R\right]}{|W| \rho\left(t_{i}\right) \rho\left(t_{j}\right)}
$$

and

$$
K_{2}(R)=\mathrm{E} \sum_{i \neq j} \frac{\mathbf{1}\left[x_{i} \in W,\left\|x_{i}-x_{j}\right\| \leq v\left(t_{i}+t_{j}\right),\left\|x_{i}-x_{j}\right\| \leq R\right]}{|W| \rho\left(t_{i}\right) \rho\left(t_{j}\right)}
$$

where E $\cdots$ denotes expectation with respect to the Johnson-Mehl process. In (19), the condition $v\left(t_{i}+\right.$ $\left.t_{j}\right)<\left\|x_{i}-x_{j}\right\|$ means that $\left(x_{i}, t_{i}\right)$ and $\left(x_{j}, t_{j}\right)$ are independent events, while in $(20)$, the condition $\left\|x_{i}-x_{j}\right\| \leq v\left(t_{i}+t_{j}\right)$ means that $\left(x_{i}, t_{i}\right)$ and $\left(x_{j}, t_{j}\right)$ are dependent events, cf. (3).

The intuitive interpretation of $K_{1}$ and $K_{2}$ can be expressed in terms of the intensity re-weighted measure $\Psi_{1 / \rho}$ defined as the random diffuse measure with events given by the points in $\Psi$, where the 
mass at $\left(x_{i}, t_{i}\right) \in \Psi$ is $1 / \rho\left(t_{i}\right)$ : if $(x, s)$ is a 'uniformly selected' event of $\Psi_{1 / \rho}$, then $K_{1}(R)$ is the expected number of independent events $(y, t)$ of $\Psi_{1 / \rho}$ with $\|x-y\| \leq R$, and $K_{2}(R)$ is the expected number of dependent events $(y, t)$ of $\Psi_{1 / \rho}$ with $\|x-y\| \leq R$. Furthermore,

$$
K_{1}(R)+K_{2}(R)=\mathrm{E} \sum_{i \neq j} \frac{\mathbf{1}\left[x_{i} \in W,\left\|x_{i}-x_{j}\right\| \leq R\right]}{|W| \rho\left(t_{i}\right) \rho\left(t_{j}\right)}
$$

is a limiting case of the space-time $K$-inhomogeneous function (Gabriel \& Diggle 2009).

Let $\sigma_{d}=2 \pi^{d / 2} / \Gamma(d / 2)$ be the surface area of the unit ball in $\mathbb{R}^{d}$. Using (15) and assuming that $\kappa>0$, we obtain

$$
K_{1}(R)=\frac{\sigma_{d}}{2 v^{2}(d+2)} R^{d+2}
$$

while

$$
\begin{aligned}
K_{2}(R) & =\sigma_{d} \int_{0}^{\infty} \int_{0}^{\infty} \int_{0}^{\infty} \mathbf{1}[v|s-t|<r \leq v(s+t), r \leq R] \\
& \times \exp \left(-\int_{0}^{(s+t-r / v) / 2} \kappa(u) V_{\cap}(r, s-u, t-u) \mathrm{d} u\right) \mathrm{d} r \mathrm{~d} s \mathrm{~d} t
\end{aligned}
$$

is more complicated to evaluate. Note that $K_{1}$ does not depend on $\kappa$ but only on the value of $v$, so in this sense $K_{1}$ is just a functional summary statistic for the (general) Johnson-Mehl model and how $v$ has been specified (or estimated). On the other hand, $K_{2}$ depends on both $\kappa$ and $v$.

Finally, for non-parametric estimation of $K_{1}$ and $K_{2}$, for $x \in W$ and $r>0$, let $w(x, r)$ denote Ripley's isotropic edge correction factor given by $1 / \sigma_{d}$ times the surface area of $W$ intersected by the boundary of $b(x, r)$ (Ripley 1976; Ripley 1977). Using (15), a straightforward calculation shows that

$$
\widehat{K}_{1}(R)=\sum_{i \neq j} \frac{\mathbf{1}\left[x_{i} \in W, x_{j} \in W, v\left(t_{i}+t_{j}\right)<\left\|x_{i}-x_{j}\right\| \leq R\right]}{|W| \rho\left(t_{i}\right) \rho\left(t_{j}\right) w\left(x_{i},\left\|x_{i}-x_{j}\right\|\right)}
$$

is an unbiased estimator of $K_{1}(R)$, and

$$
\widehat{K}_{2}(R)=\sum_{i \neq j} \frac{\mathbf{1}\left[x_{i} \in W, x_{j} \in W,\left\|x_{i}-x_{j}\right\| \leq v\left(t_{i}+t_{j}\right),\left\|x_{i}-x_{j}\right\| \leq R\right]}{|W| \rho\left(t_{i}\right) \rho\left(t_{j}\right) w\left(x_{i},\left\|x_{i}-x_{j}\right\|\right)}
$$

is an unbiased estimator of $K_{2}(R)$. In (23) we have omitted in the indicator function the condition that $v\left|t_{i}-t_{j}\right|<\left\|x_{i}-x_{j}\right\|$, since this condition is satisfied almost surely. In practice we need to plug-in an estimate of $\rho$ in (22)-(23) as further discussed in Section 4.1. As illustrated in Section 4.1.2, since the nuclei of dependent events are in sense closer than the nuclei of independent events, we should consider results for $K_{2}(R)$ for smaller values of $R$, and results for $K_{1}(R)$ for not too small values of $R$. 


\subsection{Functional summary statistics based on nuclei-boundary distances of the Johnson-Mehl tessellation}

In this section we define functional summary statistics based on the nuclei-boundary distances of the Johnson-Mehl cells, and specified in terms of so-called typical Johnson-Mehl cell. As in Section 3.1.2, we let $W \subset \mathbb{R}^{d}$ denote a bounded observation window of Lebesgue measure $|W|>0$, and assume that a realization of $\Psi_{W \times[0, \infty)}$ is observed.

First, note that with probability one, each Johnson-Mehl cell $C_{i}$ is compact and its nucleus $x_{i}$ is an interior point of $C_{i}$ (Møller 1992). Recall that $C_{i}$ has its nucleus $x_{i}$ as a starting point for growth, cf. (1). The spatial point process of nuclei is stationary and isotropic, with intensity

$$
\zeta=\int_{0}^{\infty} \rho(t) \mathrm{d} t=\int_{0}^{\infty} \exp \left(-\int_{0}^{t} \omega_{d}[v(t-s)]^{d} \kappa(s) \mathrm{d} s\right) \kappa(t) \mathrm{d} t
$$

Henceforth we assume that $0<\zeta<\infty$. This is satisfied for models M1 and M2, where for model M1 we obtain

$$
\zeta=\frac{\frac{\alpha}{\beta+d} \Gamma\left(\frac{\beta}{\beta+d}\right)}{\left[\alpha \omega_{d} v^{d} B(\beta, d+1)\right]^{\beta /(\beta+d)}}
$$

by combining (7) and (24), while for model M2 we may calculate $\zeta$ by numerical methods using (8)-(9) and (24).

Second, consider the typical Johnson-Mehl cell $C_{o}$ as defined in Appendix C. Intuitively, $C_{o}$ follows the conditional distribution of a Johnson-Mehl cell given that its nucleus is located at an arbitrary fixed point in $\mathbb{R}^{d}$, which for specificity we let be the origin $o$, say. Let $R_{o}$ denote the shortest distance from $o$ to the boundary of $C_{o}$, and define $S_{o}$ such that $R_{o} S_{o}$ is the longest distance from $o$ to the boundary of $C_{o}$. Note that $S_{o}$ is a shape characteristic of $C_{o}$, where $0 \leq S_{o} \leq 1$ and large values of $S_{o}$ corresponds to a nearly spherical shape. Particularly, if $d=1$, then either $C_{o}=\left[-R_{o}, R_{o} S_{o}\right]$ or $C_{o}=\left[-R_{o} S_{o}, R_{o}\right]$, so except for a sign $C_{o}$ is determined by $R_{o}$ and $S_{o}$. Similarly, for $\left(x_{i}, t_{i}\right) \in \Psi$, let $R_{i}$ respective $R_{i} S_{i}$ denote the shortest respective longest distance from $x_{i}$ to the boundary of $C_{i}$. Then, as noticed in Appendix C, the joint distribution of $R_{o}$ and $S_{o}$ is given as follows: for any Borel set $F \subseteq[0, \infty) \times[0,1]$,

$$
\begin{aligned}
& \zeta|W| \mathrm{P}\left(\left(R_{o}, S_{o}\right) \in F\right)= \\
& \quad \mathrm{E} \sum_{\left(x_{i}, t_{i}\right) \in \Psi_{W \times[0, \infty)}} \mathbf{1}\left[\left(R_{i}, S_{i}\right) \in F \text { and } T_{j}\left(x_{i}\right)>t_{i} \text { for all }\left(x_{j}, t_{j}\right) \in \Psi \backslash\left\{\left(x_{i}, t_{i}\right)\right\}\right] .
\end{aligned}
$$

Third, denote $D, E, F$ the distribution function of $R_{o}, S_{o},\left(R_{o}, S_{o}\right)$, respectively. Ignoring edge effects, 
a ratio unbiased non-parametric estimator of $D$ is by (25) given by

$$
\widehat{D}(R)=\sum_{\left(x_{i}, t_{i}\right) \in \Psi_{W \times[0, \infty)}} \frac{\mathbf{1}\left[R_{i} \leq R \text { and } T_{j}\left(x_{i}\right)>t_{i} \text { for all }\left(x_{j}, t_{j}\right) \in \Psi_{\left.W \times[0, \infty) \backslash\left\{\left(x_{i}, t_{i}\right)\right\}\right]}^{\hat{\zeta}|W|}\right.}{|W|}
$$

where $\hat{\zeta}$ is the natural unbiased estimate of $\zeta$ given by the number of nuclei in $\Psi_{W \times[0, \infty)}$ divided by $|W|$.

In a similar way we derive ratio unbiased non-parametric estimators $\widehat{E}$ and $\widehat{F}$ for $E$ and $F$, respectively. In this paper, the summary statistics $D$ and $E$ have been used.

Finally, we remark that the theoretical functions $D, E, F$ are rather complicated to evaluate (see Appendix C). This is in line with the fact that the known probabilistic results for the Johnson-Mehl tessellation concern mainly first order properties of $C_{o}$ (such as its mean volume, mean surface area, etc.) and a few second order properties of $C_{o}$ (Gilbert 1962), cf. the references mentioned in Section 1.2. Even for the spatial point process of nuclei, apart from knowing its intensity, results are rare, cf. Appendix B.

\section{Examples when $d=1$}

Throughout this section, $d=1, W=[0,1]$, and we consider datasets modelled as $n \gg 1$ independent realizations ('replications') of $\Psi_{W}^{\text {app }}$, cf. (10), where parametric models have been fitted by maximum likelihood as described in Section 2.4. Section 4.1 assesses the quality of the summary statistics proposed in Sections 3.1-3.2 by a simulation study, while Section 4.2 applies the summary statistics for the analysis of a real dataset. Simulation procedures under models M1 and M2 are described in Appendix D.

We use the generic notation $T$ for any of the functional summary statistics $K_{1}, K_{2}, D$, and $E$. Given a dataset, denote $\widehat{T}_{j}$ the non-parametric estimate of $T$ based on the $j$ th replicate, $j=1, \ldots, n$. As the final non-parametric estimate of $T$, we use the average

$$
\bar{T}(R)=\sum_{j=1}^{n} \widehat{T}_{j}(R) / n
$$

Moreover, for a given 'fitted model' and for each $R$-value, we estimate $99 \%$ and $1 \%$ envelopes by the largest and smallest value of $\bar{T}(R)$-values as calculated from 99 further simulations of $n$ replications of $\Psi_{W}^{\text {app }}$ under the fitted model, where after each simulation, since $\bar{T}$ depends on the parameter estimates of the parametric model, we reestimate the parameters. Then $\bar{T}(R)$ as based on the data is above or below these simulated envelopes with approximate probability $2 \%$. This envelope method is common when analyzing spatial and spatio-temporal point patterns, see Møller \& Ghorbani (2012) and the references therein. From the 99 further simulations we also calculate the average of the corresponding $99 \bar{T}(R)$-values and compare this with $\bar{T}(R)$ as based on the data. 
In the case $T=K_{1}$, we have the theoretical expression $K_{1}(R)=R^{3} /\left(3 v^{2}\right)$, cf. (21). Therefore, making the transformation

$$
\bar{M}(R)=\left(3 \hat{v}^{2} \bar{K}_{1}(R)\right)^{1 / 3}-R
$$

where $v$ has been replaced by its estimated value based on the data (or the simulated data when we are calculating envelopes), we expect $\bar{M}$ to be close to zero if the Johnson-Mehl model with $v$ estimated by its MLE (or by other means as exemplified in Section 4.1) is fitting well. Since we have a large number of replications, we also investigate the comparison of the theoretical $K_{1}$-function with $\bar{K}_{1}$ and bounds based on the central limit theorem (CLT). For example, for each number $R>0$, an approximate $98 \%$ CLT-based interval is given by $\bar{K}_{1}(R) \pm 2.326 \sqrt{s(R)^{2} / n}$ where $s(R)^{2}$ is the empirical variance obtained from $\widehat{K}_{1, j}(R), j=1, \ldots, n$ based on the data. Using the transformation (26), we thereby obtain a pointwise (approximate) 98\% CLT-based interval which we compare with the theoretical $M$-function (i.e. the zero-function).

\subsection{Simulation study}

\subsubsection{Setting}

In accordance with the neurotransmitters dataset analyzed in Section 4.2, we consider a simulated dataset consisting of $n=746$ replications of $\Psi_{W}^{\text {app }}$ under model 2 with parameter values given by the maximum likelihood estimates (MLEs) obtained in Chiu et al. (2003), i.e. $\hat{v}=0.018, \hat{\alpha}=1.29, \hat{\gamma}=13.3$, and

$\hat{\beta}=5.36$. In the sequel we refer to this simulated dataset as the 'first simulated dataset' and think of this as our 'data'.

We fit three different models to the first simulated dataset:

(i) The 'correctly specified model', which is model M2 with parameter values given by the MLEs $\hat{v}=0.018, \hat{\alpha}=1.32, \hat{\gamma}=13.32$, and $\hat{\beta}=5.35$.

(ii) The 'wrongly specified model 1', which is the reduced model M2 with $\beta=1$ and the other parameter values given by the MLEs $\hat{v}=0.018, \hat{\alpha}=1.32$, and $\hat{\gamma}=2.47$.

(iii) The 'wrongly specified model 2', which is model M2 with a smaller estimate for $v$ than the MLE, namely $\hat{v}=0.009$ while the other parameter values are given by the MLEs $\hat{\alpha}=1.32, \hat{\gamma}=13.33$, and $\hat{\beta}=5.34$.

The MLEs in (i) are close to the true parameter values. Comparing (i) and (ii), the MLEs for $\alpha$ are effectively equal while the MLEs for $\gamma$ are very different. From its definition, the MLE $\hat{v}$ used in (i)-(ii) 
is an overestimate. A simulation study in Chiu et al. (2000), but for another setting than ours, indicated that the MLE $\hat{v}$ is not seriously biased. We therefore also consider the case (iii), where $\hat{v}=0.009$ is half of the value of the MLE. Note that the MLEs for the other parameters are nearly the same in (i) and (iii). For each of the three cases, we estimate the intensity function $\rho$ by (8) with the parameter values as specified in (i)-(iii), and this estimate of $\rho$ is then used in (22)-(23) to obtain the estimates $\widehat{K}_{1, j}$ and $\widehat{K}_{2, j}$.

\subsubsection{Results}

The left panel of Figure 2 shows the theoretical $M$-function (the zero-line shown as a dot-dashed line) together with the non-parametric estimate $\bar{M}$ and the pointwise $98 \%$ CLT-based confidence intervals (dotted lines) for the $M$-function based on the first simulated dataset and $v$ is estimated by the MLE (i.e. as for the correctly specified model and for the incorrectly specified model 1). As expected, except for the larger $R$-values, $\bar{M}(R)$ is approximately zero and the confidence intervals become wider as $R$ increases. As noticed at the end of Section 3.1.2, we should not consider results for $K_{1}(R)$ for too small values of $R$. For $R$-values larger than about 0.05 , the confidence intervals in Figure 2 contain zero as we might expect, but for $R<0.05$ we may question the value of the results. In particular, for smaller $R$-values ( $R$ less than about 0.01), $\bar{M}(R)=R$ since there are no pairs of independent events at this scale; further simulations confirmed that this happens frequently, cf. Figure 3 (to be discussed later). Furthermore, in Figure 2, the mid panel is similar to the left panel but uses the estimate of $v$ under the incorrectly specified model 2. In this plot $\bar{M}$ and the pointwise 98\% CLT-based confidence intervals are (mostly) below zero. So the overall conclusion is that Figure 2 indicates no clear lack of fit for the Johnson-Mehl model when estimating $v$ by the MLE while there is a poor fit when estimating $v$ by half of the MLE.

In Figures 3-5, the top left panels show the estimate $\bar{M}$ for the first simulated dataset (solid line), the theoretical $M$-function (dot-dashed line), and the average and pointwise $98 \%$ envelopes (dashed lines) obtained from $\bar{M}$-functions calculated from 99 simulations under the correctly specified model (Figure 3), the wrongly specified model 1 (Figure 4) and the wrongly specified model 2 (Figure 5), respectively. Furthermore, the top right panels are as the top left panels but concern $K_{2}$ and without showing the theoretical $K_{2}$-function. The bottom panels show $\bar{D}$ (left) and $\bar{E}$ (right) for the first simulated dataset (solid lines) together with the average and pointwise $98 \%$ envelopes (dashed lines) obtained from $\bar{D}$-functions (left) and $\bar{E}$-functions (right) calculated from 99 simulations under the correctly specified model (Figure 3), the wrongly specified model 1 (Figure 4), and the wrongly specified model 2 (Figure 5), respectively. The plots in Figures 3-5 taken together indicate a satisfactory fit for the correctly specified 

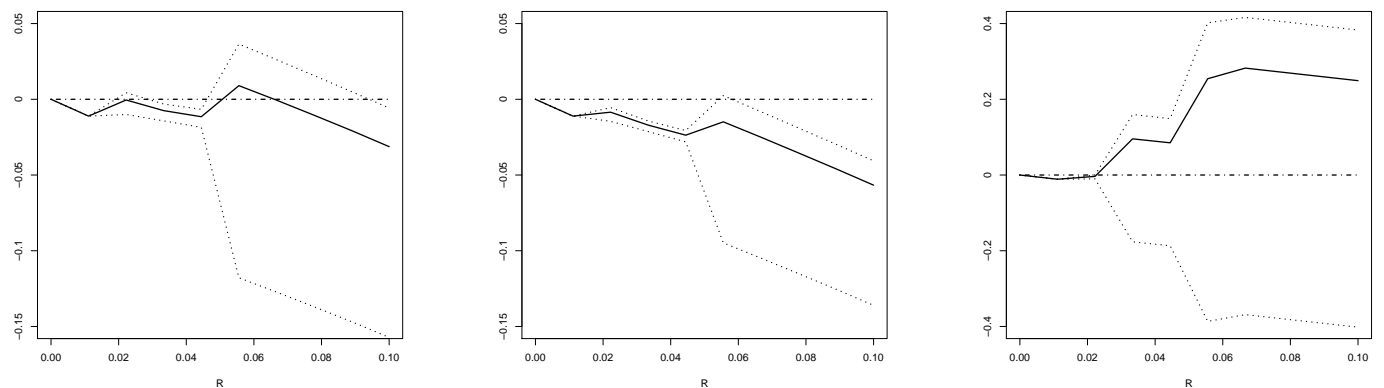

Figure 2: Left: theoretical $M$-function (dot-dashed line), $\bar{M}$ (solid line), and pointwise $98 \%$ CLT-based confidence intervals (dotted lines) for the $M$-function based on the first simulated dataset and when $v$ is estimated by the MLE. Mid: as the left plot but when $v$ is estimated by half of the MLE. Right: as the left plot but for the neurotransmitters dataset fitted in Section 4.2.

model and an unsatisfactory fit for the wrongly specified models.

Similar results were obtained when repeating the simulations a number of times. So at least for cases similar to the neurotransmitters dataset analyzed in the next section, our functional summary statistics are useful tools for checking fitted parametric Johnson-Mehl models, including the fitted model in Chiu et al. (2003).

\subsection{Neurotransmitters dataset}

This section applies our model checking procedures on the neurotransmitters dataset previously analyzed in Chiu et al. (2003) and other papers discussed below.

\subsubsection{Background}

Bennett \& Robinson (1990) suggested the following mechanism for neurotransmitters: The neuronal axon terminal at the neuromuscular junction has branches that consist of strands containing many randomly scattered sites. An action potential triggers the release of neurotransmitters to the synapse as the synaptic vesicles diffuse into the cellular membrane. Each quantum released is assumed to cause the release of an inhibitory substance which diffuses along the terminal at a constant rate preventing further releases in the inhibited region. Thus, some potential releases have been prohibited (for more details, see Bennett \& Robinson (1990)).

Different neurotransmitters datasets (provided by Professor M. R. Bennett, Neurobiology Research 

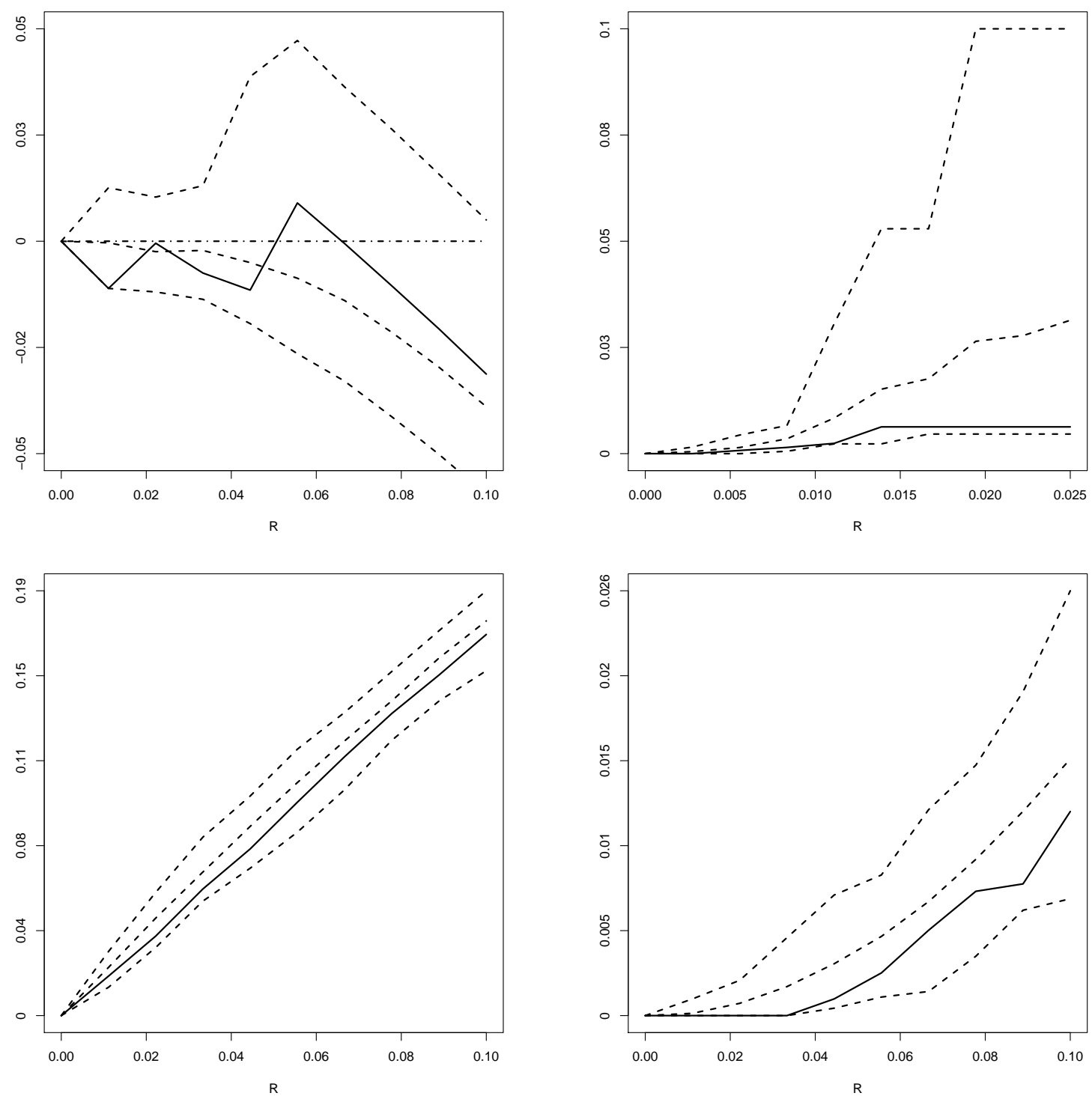

Figure 3: Correctly specified model. Top left panel: $\bar{M}$ (solid line), theoretical $M$-function (dot-dashed line), and average and pointwise $98 \%$ envelopes calculated from 99 further simulations under the correctly specified model (dashed lines). Top right panel: as the top left panel but for the $K_{2}$-function and without showing the theoretical $K_{2}$-function. Bottom panels: as the top right panel but for the $D$-function (left) and the $E$-function (right). 

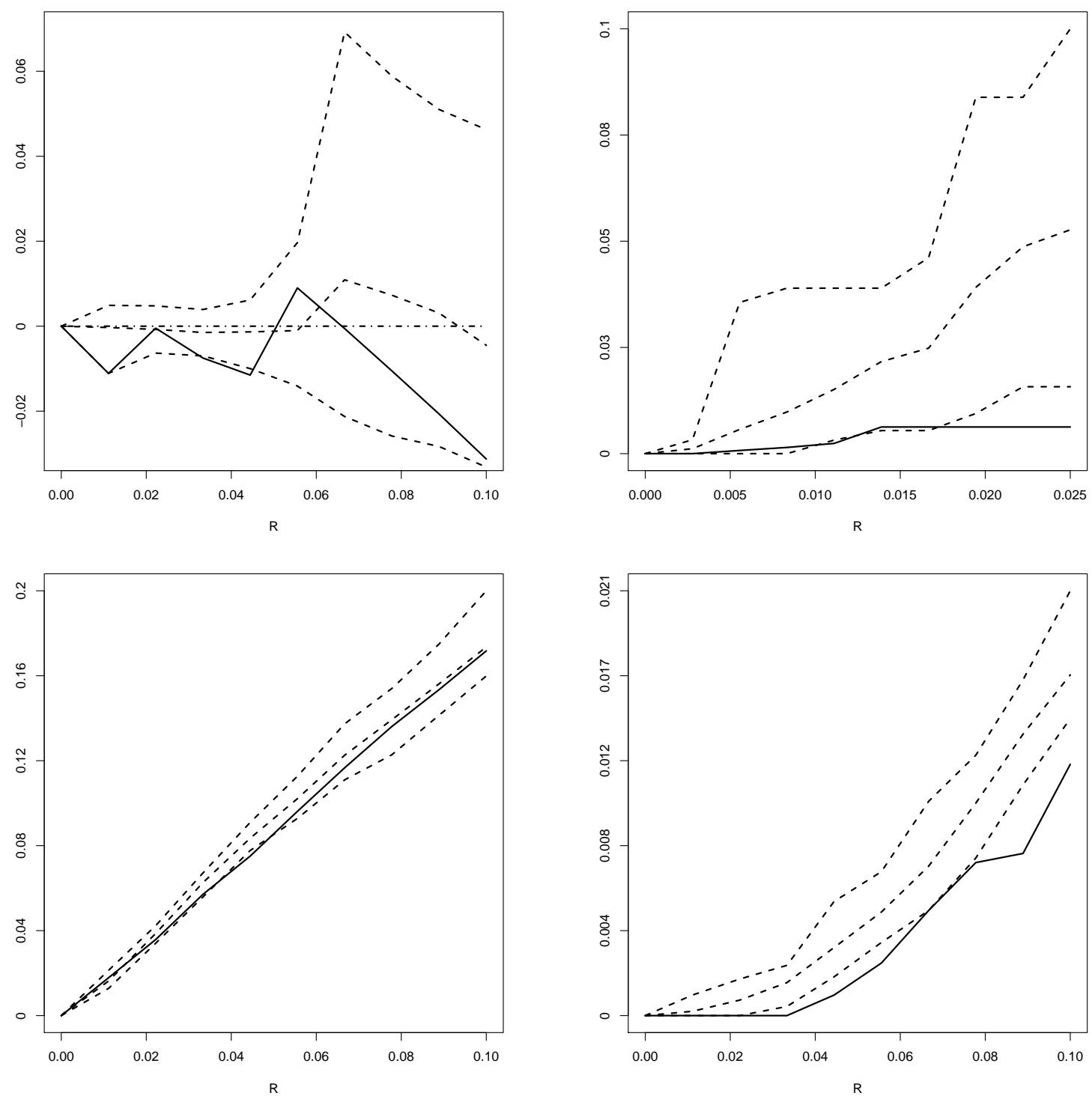

Figure 4: Wrongly specified model 1 . Top left panel: $\bar{M}$ (solid line), theoretical $M$-function (dot-dashed line), and average and pointwise $98 \%$ envelopes calculated from 99 simulations under the wrongly specified model 1 (dashed lines). Top right panel: as the top left panel but for the $K_{2}$-function and without showing the theoretical $K_{2}$-function. Bottom panels: as the top right panel but for the $D$-function (left) and the $E$-function (right). 

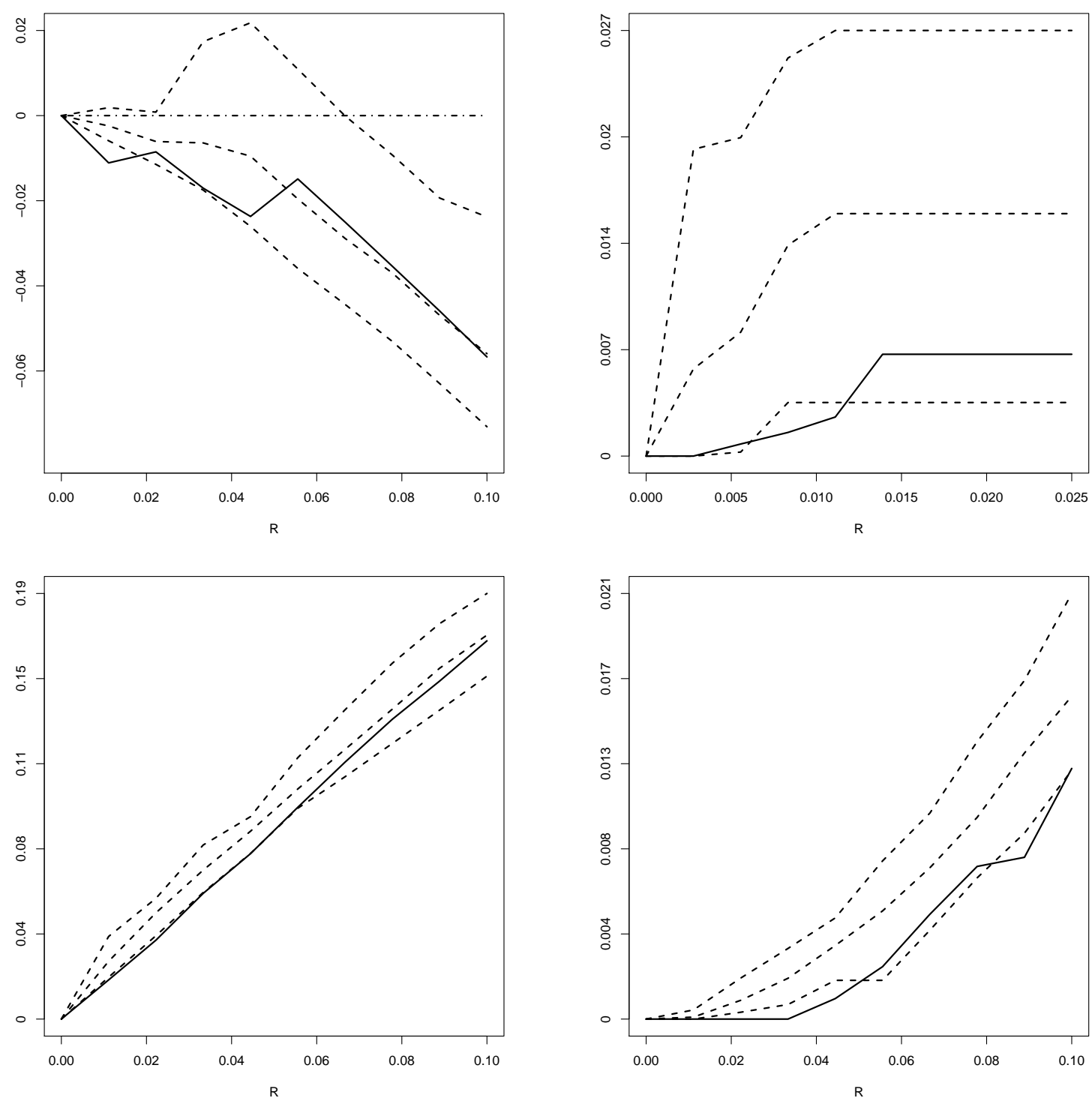

Figure 5: As in Figure 4 but for the wrongly specified model 2. 
Center, University of Sydney) have been analyzed in various ways, see Quine \& Robinson (1992), Thomson, Lavidis, Robinson \& Bennett (1995), Holst, Quine \& Robinson (1996), Chiu et al. (2000), Molchanov \& Chiu (2000), and Chiu et al. (2003). We focus on the dataset analyzed in the latter three papers. It consists of the times and the amplitudes of release of neurotransmitters in a series of 746 experiments (after omitting some experiments due to multiple amplitudes or outliers). The range of the number of releases is from 0 to 4 , and the frequencies of experiments with $0,1,2,3$, and 4 releases are 101, 387, 210,45 , and 3 , respectively. We follow the authors in using the transformations

$$
\text { nucleus }=5 / \sqrt{\text { amplitude }}, \quad \text { time }=(\text { released time }-500) / 500 .
$$

Chiu et al. (2003) fitted model M2 by maximum likelihood and obtained the MLEs $\hat{v}=0.018, \hat{\alpha}=1.29$, $\hat{\gamma}=13.3$, and $\hat{\beta}=5.36$.

\subsubsection{Extension of model M1}

Model M1 is inappropriate, since an empty realization of $\Psi_{W}^{\mathrm{app}}$ is possible under M2 but not under M1, and the neurotransmitters dataset includes in fact 101 empty realizations. We therefore extend M1 by introducing a Bernoulli variable $Z$ with parameter $\pi \in(0,1)$ such that $\mathrm{P}(Z=0)=1-\mathrm{P}(Z=1)=\pi$, $\Psi_{W}^{\mathrm{app}}=\emptyset$ if $Z=0$, and $\Psi_{W}^{\mathrm{app}}$ follows M1 if $Z=1$. Under this extension of M1, we have derived MLEs $\hat{v}=0.018, \hat{\pi}=0.135, \hat{\alpha}=0.21$, and $\hat{\beta}=0.28$. As the pair correlation function under the extended model M1 is the same as under the original model M1, the functions $K_{1}$ and $K_{2}$ are unchanged under the two models.

\subsubsection{Results}

The right panel of Figure 2 shows the theoretical $M$-function (dot-dashed line) together with the nonparametric estimate $\bar{M}$ (solid line) and the pointwise 98\% CLT-based confidence intervals (dotted lines) for the $M$-function based on the neurotransmitters dataset and when $v$ is estimated by the MLE. Ignoring the results for the smaller $R$-values (where we have very few pairs of independent events), there is no disagreement with the assumption of a Johnson-Mehl model with $v$ estimated by the MLE.

Figure 6 shows results when model M2 is fitted to the neurotransmitters dataset: the top panels show $\bar{M}$ (left) and $\bar{K}_{2}$ (right) and the bottom panels show $\bar{D}$ (left) and $\bar{E}$ (right) for the data (solid lines) together with the averages and the pointwise $98 \%$ envelopes (dashed lines) obtained from 99 simulations under the fitted model M2. Figure 7 is similar to Figure 6 but concerns the fitted extended model M1. For both fitted models, Figures 6-7 strongly indicate a poor fit. 

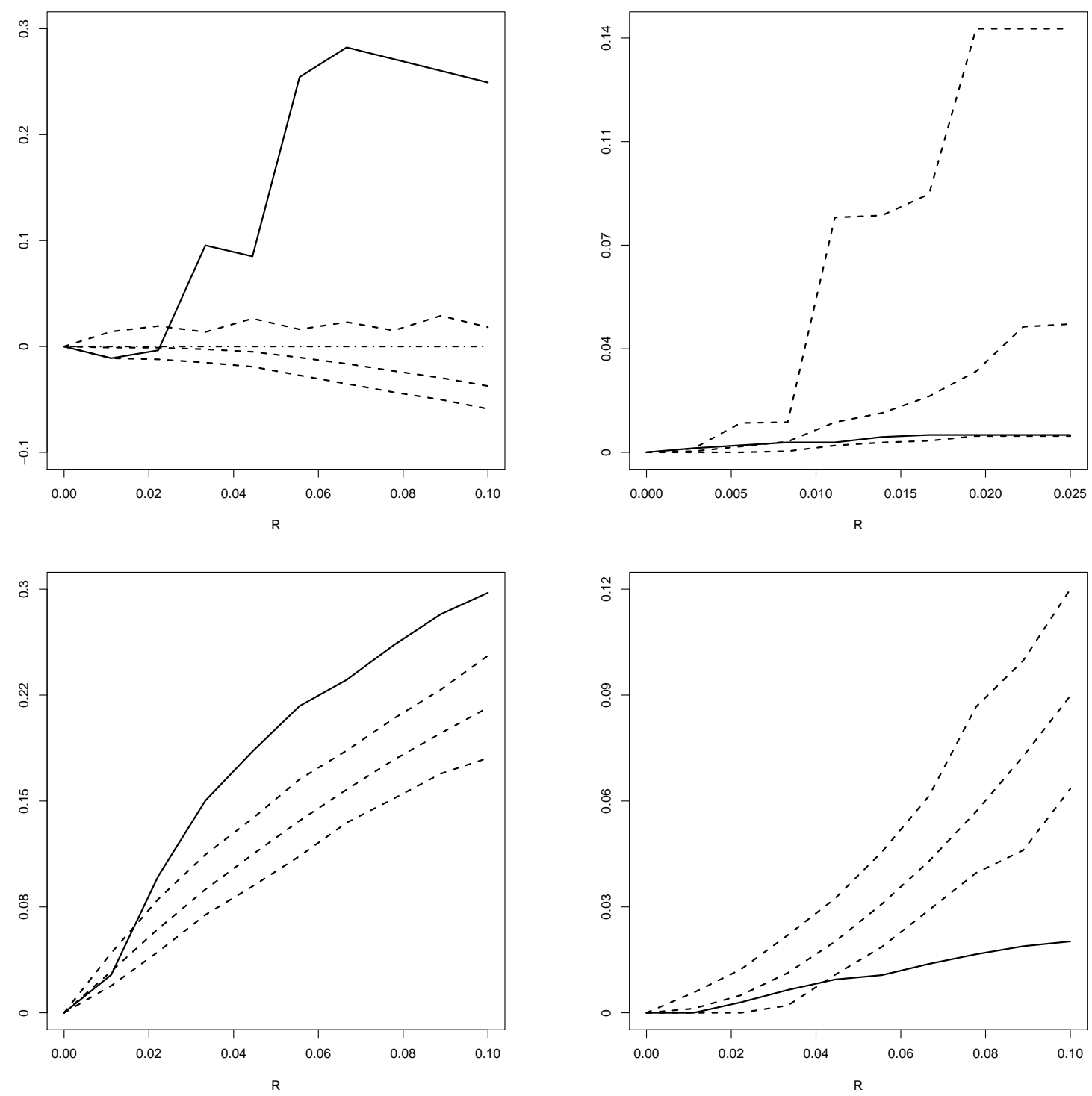

Figure 6: Results for the fitted model M2. Top left panel: $\bar{M}$ for the data (solid line), the theoretical $M$ function (dot-dashed line), and the average and pointwise $98 \%$ envelopes calculated from 99 simulations under the fitted model M2 (dashed lines). Top right panel: as top left panel but for the $K_{2}$-function and without showing the theoretical $K_{2}$-function. Bottom panels: as top right panel but for $D$-function (left) and $E$-function (right). 

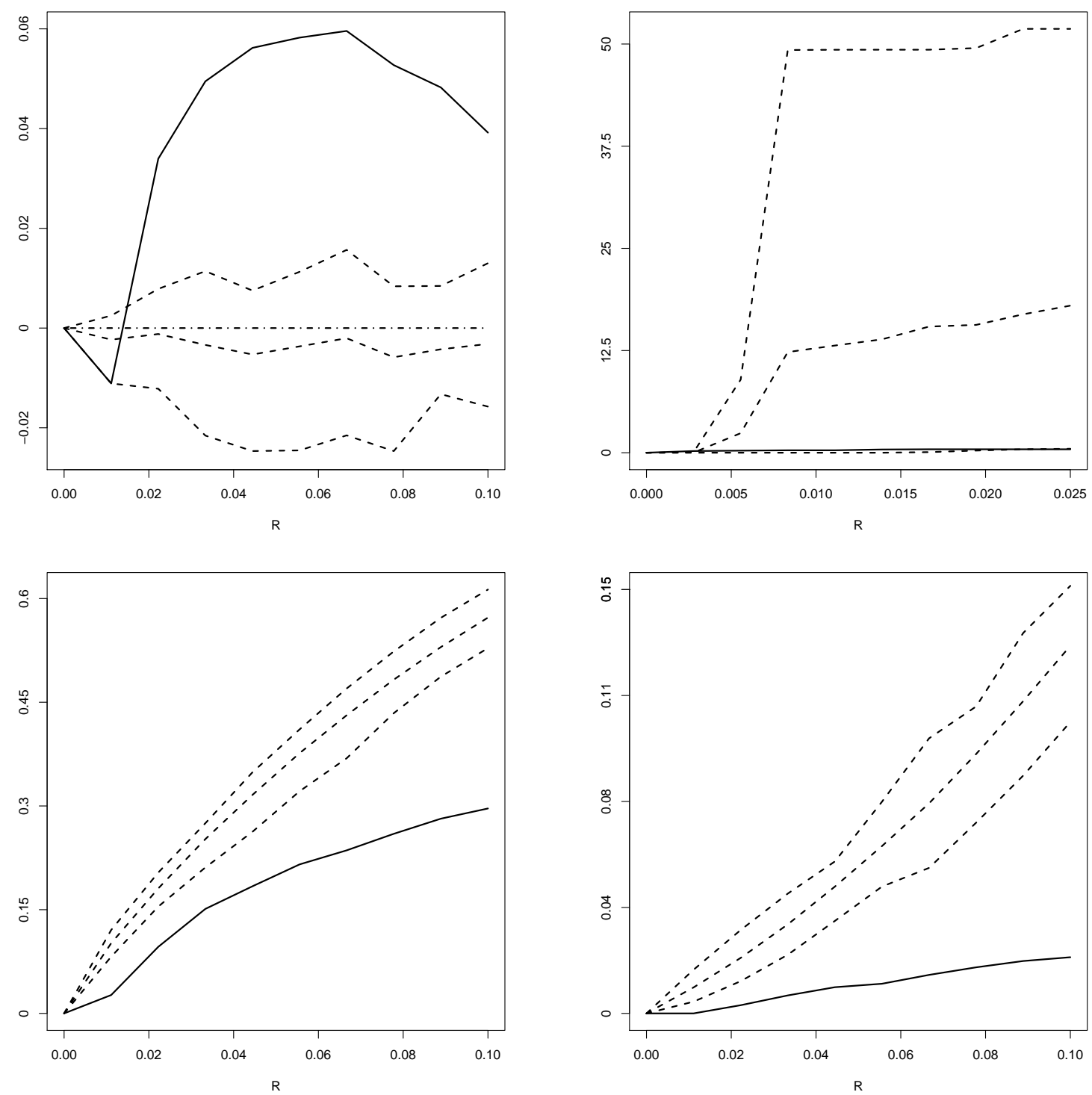

Figure 7: As Figure 6 but for the fitted extended model M1. 
Chiu et al. $(2000,2003)$ claimed that the nuclei obtained by the transformation in (27) are "roughly uniform on $[0,1]$ ", but the histogram in the top left panel of Figure 8 is clearly showing a non-uniform distribution. The Q-Q plot in the lower left panel of Figure 8 is showing that the times obtained by the transformation in (27) are not well described by the gamma distribution fitted by Chiu et al. (2003). Instead of the transformations (27), using the transformations

$$
\text { nuclei }=F((\log (\text { amplitude })-4.6) / 0.4), \quad \text { time }=(\text { released time }-523) / 500,
$$

and reestimating the parameters by maximum likelihood, we obtained a better agreement with a uniform and a gamma distribution, cf. the right panels of Figure 8. Here $F$ denotes the cumulative distribution function for the standard normal distribution, and 4.6 respective 0.4 are the average respective the standard deviation of the log-transformed amplitudes. However, plots of the functional summary statistics for this case are very similar to those in Figures 6-7 (the plots are therefore omitted here). We are therefore in doubt if the neurotransmitters dataset may be well described by a parametric Johnson-Mehl model.

\section{Acknowledgment}

Supported by the Danish Council for Independent Research - Natural Sciences, grant 12-124675, "Mathematical and Statistical Analysis of Spatial Data", and by the Centre for Stochastic Geometry and Advanced Bioimaging, funded by a grant from the Villum Foundation. We thank Sung Nok Chiu for providing the neurotransmitters dataset studied in Section 4.2.

\section{Appendix A: the volume of the intersection of two balls}

For $r>v|s-t|$ and $0 \leq u<(s+t-r / v) / 2$, combining (16)-(18), we obtain

$$
\begin{aligned}
V_{\cap}(r, s-u, t-u) & =[v(t-u)]^{2} \cos ^{-1}\left(\frac{r^{2}+[v(t-u)]^{2}-[v(s-u)]^{2}}{2 r v(t-u)}\right) & \\
& \times[v(s-u)]^{2} \cos ^{-1}\left(\frac{r^{2}+[v(s-u)]^{2}-[v(t-u)]^{2}}{2 r v(s-u)}\right) & \\
& -\frac{1}{2} \sqrt{(-r+v(s-u)+v(t-u)(r+v(s-u)-v(t-u))} & \\
& \times \sqrt{(r-v(s-u)+v(t-u)(r+v(s-u)+v(t-u))} & \text { if } d=2, \\
V_{\cap}(r, s-u, t-u) & =\frac{\pi}{12 r}(v(s+t)-2 v u-r)^{2}\left(r^{2}-3 v^{2}\left[(t-u)^{2}+(s-u)^{2}\right]\right. & \\
& \left.\left.+2 r[v(s+t)-2 u v]+6\left[v^{2}(t-u)(s-u)\right]\right)\right) & \text { if } d=3 .
\end{aligned}
$$



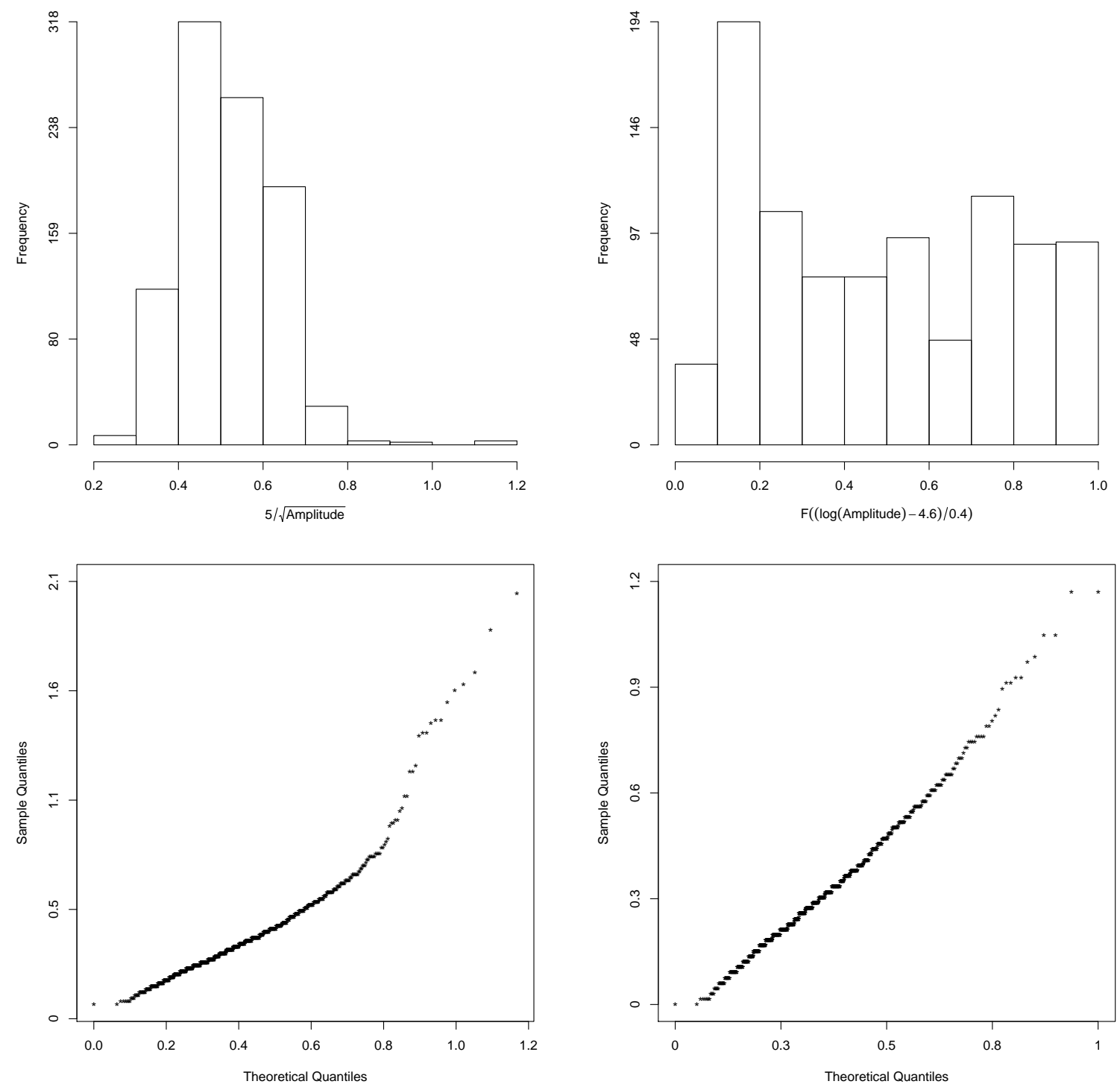

Figure 8: Top panels: histogram for the nuclei obtained by the transformation in (27) (left) or (28) (right). Bottom panels: Q-Q plot for the times obtained by the transformation in (27) (left) or (28) (right). 


\section{Appendix B: the pair correlation function for the process of nuclei}

The second-order product density for the process of nuclei is for any $x, y \in \mathbb{R}^{d}$ with $r=\|x-y\|>0$ given by $\rho^{(2)}(x, y)=\rho_{0}^{(2)}(r)$, where

$$
\rho_{0}^{(2)}(r)=\int_{0}^{\infty} \int_{0}^{\infty} \rho_{0}^{(2)}(r, s, t) \mathrm{d} s \mathrm{~d} t
$$

It is easily seen that the corresponding pair correlation function

$$
g(r)=\rho_{0}^{(2)}(r) / \zeta^{2}
$$

is less than one, which shows that the nuclei exhibit regularity. In principle $g(r)$ could be calculated using (29) and the results in Section 3.1.1, but in general we obtain some integral expressions which have to be evaluated by numerical methods. For example, for $d=1$, we obtain

$$
g(r)=\int_{0}^{\infty} \int_{0}^{\infty}\left[\kappa(s) \kappa(t) \mathbf{1}[r>v|s-t|] \exp \left(-\int_{0}^{\max \{s, t\}} \kappa(u)[v(s+t-2 u)+r] \mathrm{d} u\right)\right] \mathrm{d} s \mathrm{~d} t / \zeta^{2} .
$$

If $\kappa$ is constant (model M1 with $\beta=1$ ), a closed form expression of $g$ in terms of the cumulative distribution function of the standard normal distribution is available.

\section{Appendix C: the typical Johnson-Mehl cell}

The typical Johnson-Mehl cell $C_{o}$, or more precisely its distribution, can be defined as follows (Møller 1992).

The nucleus of $C_{o}$ is given by an arbitrary fixed point $o$, let us say the origin of $\mathbb{R}^{d}$. The time where the nucleus $o$ starts to grow is a random variable $T_{o}$ which is independent of $\Phi$ and has density $\rho / \zeta$. For $t>0$, let

$$
C(o, t ; \Psi)=\left\{y \in \mathbb{R}^{d}: T((o, t), y) \leq T_{i}(o) \text { for all }\left(x_{i}, t_{i}\right) \in \Psi\right\}
$$

denote the set of those points first reached by $o$ when considering all the growing nuclei from the JohnsonMehl process $\Psi$. By definition, the conditional distribution of $C_{o}$ given $T_{o}=t$ is the same as the conditional distribution of $C(o, t ; \Psi)$ given that $T_{i}(o)>t$ for all $\left(x_{i}, t_{i}\right) \in \Psi$, i.e. when $o$ is not thinned by $\Psi$. In other words, considering the Johnson-Mehl tessellation generated by both $\left(o, T_{o}\right)$ and $\Phi, C_{o}$ is the cell with nucleus $o$ and as a matter of fact, with probability one, $C_{o}$ is compact and $o$ is an interior point of $C_{o}$. 
Combining this definition with the Slivnyak-Mecke formula (Mecke 1967; Møller \& Waagepetersen 2004), we obtain that (25) holds for any Borel set $W$. Furthermore,

$$
D(r)=1-\iint P(\Phi \cap H(t, r)=\emptyset) \kappa(t) \mathrm{d} x \mathrm{~d} t / \zeta, \quad r>0,
$$

where

$$
\begin{aligned}
H(t, r) & =\left\{(y, u) \in \mathbb{R}^{d} \times[0, t]:\|y\| \leq 2 r+v(t-u)\right\} \\
& \cup\left\{(y, u) \in \mathbb{R}^{d} \times(t, t+r / v]: v(t-u) \leq\|y\| \leq 2 r+v(t-u)\right\} .
\end{aligned}
$$

For instance, for model M1 and $d=1$, we obtain

$$
D(r)=1-\int \exp \left(\frac{-2 \alpha}{\beta}\left(2 r(t+r / v)^{\beta}+\frac{v}{\beta+1} t^{\beta+1}\right)\right) \alpha t^{\beta-1} \mathrm{~d} t / \zeta .
$$

This illustrates that $D, E, F$ are given by integral expressions which may only be evaluated by numerical methods.

\section{Appendix D: simulation procedure}

First, we consider simulation of $\Psi_{W}^{\mathrm{app}}$ under the extension of M1 given in Section 4.2.1 when $d=1$. This boils down to how to simulate under the original model M1, where we use the following inversion method.

Suppose $u_{1}, u_{2}, \ldots$ are independent realizations of exponentially distributed random variables with unit rate. Then $u_{1}, u_{1}+u_{2}, \ldots$ are forming a realization of a unit rate Poisson process on $[0, \infty)$. The integrated intensity

$$
\Lambda(t) \equiv \int_{0}^{t} \alpha s^{\beta-1} \mathrm{~d} s=\alpha t^{\beta} / \beta, \quad t \geq 0,
$$

has inverse $\Lambda^{-1}(u)=(\beta u / \alpha)^{1 / \beta}$, so $t_{1}=\Lambda^{-1}\left(u_{1}\right), t_{2}=\Lambda^{-1}\left(u_{1}+u_{2}\right), \ldots$ are forming a realization of a Poisson process on $[0, \infty)$ with intensity function $\alpha t^{\beta-1}$. To each $t_{i}$, attach a point $x_{i} \in W$, where these points are independent realizations of uniformly distributed random variables on $W$ which in turn are independent of the exponentially distributed random variables. Then $\left\{\left(x_{1}, t_{1}\right),\left(x_{2}, t_{2}\right), \ldots\right\}$ is a realization of the Poisson process $\Phi_{W \times[0, \infty)}$ under M1.

Now, as in Section 4 , let $W=[0,1]$. Then we simulate a realization of $\Psi_{W}^{\text {app }}$ under M1 as follows. We start by generating $\left(x_{1}, t_{1}\right)$ and $t_{2}$ and by setting $\Psi_{W}^{\text {app }}=\left\{\left(x_{1}, t_{1}\right)\right\}$. We stop if $t_{2} \geq T$, where $T$ is the time $W$ is covered when $x_{1}$ is growing spherically with speed $v$, i.e. $T=\max \left\{T_{1}(0), T_{2}(1)\right\}$. If $t_{2}<T$, then we have to generate $x_{2}$ and to check if $\left(x_{2}, t_{2}\right)$ is thinned by $\left(x_{1}, t_{1}\right)$. If it is not, i.e. if $T_{1}\left(x_{2}\right)>t_{2}$, then we include $\left(x_{2}, t_{2}\right)$ in $\Psi_{W}^{\text {app }}$ and update $T$ by the time at which $W$ is covered when $x_{1}$ and $x_{2}$ are 
growing spherically with speed $v$, i.e. $T=\max \left\{T_{1}(0), T_{2}(1), T_{1,2}\right\}$, where $T_{1,2}=\left(t_{1}+t_{2}+\left(x_{2}-x_{1}\right) / v\right) / 2$ is the time at which the rays growing from $x_{1}$ and $x_{2}$ are meeting. If $t_{3} \geq T$ then we stop, and else we continue in the same way until we obtain the first time where $t_{i} \geq T$ (with probability one, this happens at some point).

Next, to simulate under model M2 within $W=[0,1]$, we first generate the primary space-time Poisson process $\Phi$ restricted to $[0,1] \times[0, \infty)$ : this is straightforward as the number of events is Poisson distributed with mean $\alpha$, the $x_{i}$ are uniformly distributed on $W$, the $t_{i}$ are gamma distributed with shape parameter $\beta$ and inverse scale parameter $\gamma$, and all the $x_{i}$ and all the $t_{i}$ are independent. Second, using the thinning mechanism described in Section 1.1, we obtain the events of $\Psi_{W}^{\mathrm{app}}$.

\section{References}

Baddeley, A., Møller, J. \& Waagepetersen, R. (2000). Non- and semi-parametric estimation of interaction in inhomogeneous point patterns, Statistica Neerlandica 54: 329-350.

Bennett, M. R. \& Robinson, J. (1990). Probabilistic secretion of quanta from nerve terminals at synaptic sites on muscle cells: Non-uniformity, autoinhibition and the binomial hypothesis, Proceedings of the Royal Society of London. Series B, Biological Sciences 239: 1049-1052.

Boots, B., Okabe, A. \& Thomas, R. (eds) (2003). Modelling Geographical Systems: Statistical and Computational Applications, Kluwer Academic Publishers, Dordrecht.

Chiu, S. N. (1995). Limit theorems for the time of completion of Johnson-Mehl tessellations, Advances in Applied Probability 27: 889-910.

Chiu, S. N., Molchanov, I. S. \& Quine, M. P. (2003). Maximum likelihood estimation for germinationgrowth processes with application to neurotransmitters data, Journal of Statistical Computation and Simulation 73: 725-732.

Chiu, S. N., Quine, M. P. \& Stewart, M. (2000). Nonparametric and parametric estimation for a linear germination-growth model, Biometrics 56: 755-760.

Cowan, R., Chiu, S. N. \& Holst, L. (1995). A limit theorem for the replication time of a DNA molecule, Journal of Applied Probability 32: 296-303.

Daley, D. J. \& Vere-Jones, D. (2003). An Introduction to the Theory of Point Processes. Volume I: Elementary Theory and Methods, second edn, Springer-Verlag, New York. 
Frost, H. J. \& Thompson, C. V. (1987). The effect of nucleation conditions on the topology and geometry of two-dimensional grain structures, Acta Metallurgica et Materialia 35: 529-540.

Frost, H. J., Thompson, C. V. \& Walton, D. T. (1992). Simulation of thin film grain structures-II. Abnormal grain growth, Acta Metallurgica et Materialia 40: 779-793.

Gabriel, E. \& Diggle, P. J. (2009). Second-order analysis of inhomogeneous spatio-temporal point process data, Statistica Neerlandica 63: 43-51.

Gelfand, A. E., Diggle, P. J., Guttorp, P. \& Fuentes, M. (2010). Handbook of Spatial Statistics, CRC Press, Boca Raton.

Gilbert, E. N. (1962). Random subdivisions of space into crystals, Annals of Mathematical Statistics 33: $958-972$.

Holst, L., Quine, M. P. \& Robinson, J. (1996). A general stochastic model for nucleation and linear growth, Annals of Applied Probability 6: 903-921.

Horálek, V. (1988). A note on the time-homogeneous Johnson-Mehl tessellation, Advances of Applied Probability 20: 684-685.

Horálek, V. (1990). ASTM grain-size model and related random tessellation models, Materials Characterization 25: 263-284.

Johnson, W. A. \& Mehl, R. F. (1939). Reaction kinetics in processes of nucleation and growth, Transactions of the American Institute of Mining and Metallurgical Engineers 135: 416-458.

Kayser, K. \& Stute, H. (1989). Minimum spaning tree, Voronoi's tessellation and Johnson-Mehl diagrams in human lung carcinoma, Pathology, Research and Practice 185: 729-734.

Kenkel, N. C. (1990). Spatial competition models for plant populations, COENOSES 5: 149-158.

Kolmogorov, A. N. (1937). On the statistical theory of the crystallization of metals, Bulletin of the Academy of Sciences of the USSR, Mathematical Series 1: 355-359.

Li, S. (2011). Concise formulas for the area and volume of a hyperspherical cap, Asian Journal of Mathematics and Statistics 4: 66-70.

Mecke, J. (1967). Stationäre zufällige Maße auf lokalkompakten Abelschen Gruppen, Zeitschrift für Wahrscheinlichkeitstheorie und verwandte Gebiete 9: 36-58. 
Meijering, J. L. (1953). Interface area, edge length, and number of vertices in crystal aggregates with random nucleation, Philips Research Reports 8: 270-290.

Miles, R. E. (1972). The random subdivision of space, Supplement to Advances of Applied Probability 4: 243-266.

Molchanov, I. S. \& Chiu, S. N. (2000). Smoothing techniques and estimation methods for nonstationary Boolean models with applications to coverage processes, Biometrika 87: 265-283.

Møller, J. (1992). Random Johnson-Mehl tessellations, Advances in Applied Probability 24: 814-844.

Møller, J. (1994). Lectures on Random Voronoi Tessellations, Lecture Notes in Statistics 87, SpringerVerlag, New York.

Møller, J. (1995). Generation of Johnson-Mehl crystals and comparative analysis of models for random nucleation, Advances in Applied Probability 27: 367-383.

Møller, J. \& Ghorbani, M. (2012). Aspects of second-order analysis of structured inhomogeneous spatiotemporal point processes, Statistica Neerlandica 66: 472-491.

Møller, J. \& Waagepetersen, R. P. (2004). Statistical Inference and Simulation for Spatial Point Processes, Chapman and Hall/CRC, Boca Raton.

Okabe, A., Boots, B., Sugihara, K. \& Chiu, S. N. (2000). Spatial Tessellations. Concepts and Applications of Voronoi Diagrams, second edn, Wiley, Chichester.

Quine, M. P. \& Robinson, J. (1990). A linear random growth model, Journal of Applied Probability 27: 499-509.

Quine, M. P. \& Robinson, J. (1992). Estimation for a linear growth model, Statistics and Probability Letters 15: 293-297.

Ripley, B. D. (1976). The second-order analysis of stationary point processes, Journal of Applied Probability 13: 255-266.

Ripley, B. D. (1977). Modelling spatial patterns (with discussion), Journal of the Royal Statistical Society Series B 39: 172-212.

Stoyan, D., Kendall, W. S. \& Mecke, J. (1995). Stochastic Geometry and Its Applications, second edn, Wiley, Chichester. 
Stoyan, D. \& Stoyan, H. (1996). Estimating pair correlation functions of planar cluster processes, Biometrical Journal 38: 259-271.

Thomson, P. C., Lavidis, N. A., Robinson, J. \& Bennett, M. R. (1995). Probabilistic secretion of quanta at somatic motor-nerve terminals: The fusion-pore model, quantal detection and autoinhibition, Philosophical Transactions: Biological Sciences 349: 197-214.

Vanderbei, R. \& Shepp, L. (1988). A probabilistic model for the time to unravel a strand of DNA, Stochastic Models 4: 299-314.

Wolk, C. P. (1975). Formation of one-dimensional patterns by stochastic processes and by filamentous blue-green algae, Developmental Biology 46: 370-382. 